\section{OP0107 \\ HETEROZYGOUS MUTATIONS IN COPA ARE ASSOCIATED WITH ENHANCED TYPE I INTERFERON SIGNALLING}

Marie-Louise Frémond ${ }^{1}$, Alice Lepelley ${ }^{1}$, Carolina Uggenti ${ }^{2}$, Maria José MartinNiclos ${ }^{1}$, Marine Depp ${ }^{2}$, Vincent Bondet ${ }^{3}$, Darragh Duffy ${ }^{3}$, Gillian I Rice ${ }^{4}$, Mary Brennan ${ }^{5}$, Caroline Thumerelle ${ }^{6}$, Siham Boulisfane ${ }^{6}$, Marie Legendre ${ }^{7}$ Serge Amselem ${ }^{7}$, Thierry Molina ${ }^{8}$, Nadia Nathan ${ }^{9}$, Yanick $\mathrm{Crow}^{1,2}{ }^{1}{ }^{1} /$ magine Institute, Laboratory of Neurogenetics and Neuroinflammation, Paris, France; ${ }^{2}$ Institute of Genetics and Molecular Medicine, Centre for Genomic and Experimental Medicine, Edinburgh, United Kingdom; ${ }^{3}$ Institut Pasteur, Immunobiology of Dendritic Cells, Paris, France; ${ }^{4}$ Manchester Academic Health Science Centre, Division of Evolution and Genomic Sciences, Manchester, United Kingdom; ${ }^{5}$ Royal Hospital for Sick Children, Department of Paediatric Rheumatology, Edinburgh, United Kingdom; ${ }^{6} \mathrm{CHU}$ de Lille, Pediatrics Department, Lille, France; ${ }^{7}$ Trousseau Hospital-APHP and Sorbonne Université, Genetic Department and Inserm UMR S933, Paris, France; ${ }^{8}$ Necker Hospital-APHP, Pathology Department, Paris, France; ${ }^{9}$ RespiRare, Trousseau Hospital-APHP and Sorbonne Université, Inserm UMR S933 and Pediatric Pulmonology department and Reference Centre for Rare Lung Diseases, Paris, France

Background: Heterozygous mutations in COPA, encoding coatomer protein subunit alpha, cause an autosomal dominant inflammatory syndrome associating lung, joint and renal disease, showing some overlap with STING-associated vasculopathy with onset in infancy (SAVI). Mutations were originally described to cause endoplasmic reticulum (ER) stress and priming of a Thelper 17 response. More recently, increased transcription of interferon (IFN)-stimulated genes (ISGs) was reported in blood circulating cells of affected individuals. However, the precise pathophysiology of this disease remains unclear.

Objectives: To better decipher the mechanism of COPA syndrome.

Methods: We studied 8 patients from 3 unrelated families, each segregating a heterozygous mutation in COPA. We assessed type I IFN status by IFN $\alpha$ ultra-sensitive digital quantification in plasma, STAT1 phosphorylation and RNA expression of ISGs in whole blood from patients. In vitro assays also were performed in HEK293T and THP-1 cells to study IFN signalling in the context of COPA mutations.

Results: We observed commonalities in the lung pathology between COPA and $\mathrm{SAVI}$, as well as an IFN signature, raised levels of IFN $\alpha$ protein in the serum and phosphorylation of STAT1 in patient T cells. In a cellular model of HEK293T, phosphorylation of IRF3 and increased ISG expression were observed in cells cotransfected with wild type STING and mutant COPA plasmids. In THP-1 cells, short hairpin RNA knockdown of COPA induced IFN signalling that was abrogated in the absence of STING.

Conclusion: Our data suggest that mutations in COPA lead to constitutive activation of type I IFN signalling through STING. Based on these results, one patient has been treated with the JAK1/2 inhibitor ruxolitinib for the last 12 months. How COPA interacts with ER-resident STING remains to be investigated.

\section{REFERENCES:}

[1] Watkin et al, Nat Genet 2015;47:654-60.

[2] Volpi et al, Clin Immunol 2018;187:33-36.

Disclosure of Interests: None declared

DOI: 10.1136/annrheumdis-2019-eular.4158

THURSDAY, 13 JUNE 2019

\section{Psoriatic arthritis: old and new drugs and how to deal with them?}

\section{OP0108 DUAL NEUTRALISATION OF IL-17A AND IL-17F WITH BIMEKIZUMAB IN PATIENTS WITH ACTIVE PSA: OVERALL AND TNF-INHIBITOR-NAÏVE POPULATION RESULTS FROM A 48-WEEK PHASE 2B RANDOMISED STUDY}

Christopher T. Ritchlin ${ }^{1}$, Arthur Kavanaugh ${ }^{2}$, Joseph F. Merola ${ }^{3}$, Georg Schett ${ }^{4}$, Jose U. Scher ${ }^{5}$, Richard B. Warren ${ }^{6}$, Deepak Assudani ${ }^{7}$, Thomas Kumke ${ }^{8}$, Barbara Ink ${ }^{7}$, lain Mcinnes ${ }^{9} .{ }^{1}$ University of Rochester Medical Centre, Rochester, United States of America; ${ }^{2}$ UC San Diego School of Medicine, La Jolla, United States of America; ${ }^{3}$ Harvard Medical School, Brigham and Women's Hospital, Boston, United States of America; ${ }^{4}$ Friedrich Alexander University ErlangenNurnberg, Erlangen, Germany; ${ }^{5}$ NYU Langone Medical Center, Department of Medicine, New York, United States of America; ${ }^{6}$ The Dermatology Centre, Salford Royal NHS Foundation Trust, The University of Manchester, Manchester, United Kingdom; ${ }^{7}$ UCB Pharma, Slough, United Kingdom; ${ }^{8}$ UCB Pharma, Monheim am Rhein, Germany; ${ }^{9}$ University of Glasgow, Glasgow, United Kingdom

Background: IL-17F shares structural homology and pro-inflammatory function with IL-17A. Preclinical and early clinical data support neutralisation of
IL-17F, in addition to IL-17A, as a novel targeting approach in psoriatic disease.

Objectives: The objective of this Phase 2b study (NCT02969525) was to assess the dose response, long-term efficacy and safety of bimekizumab (BKZ), a mAb that potently and selectively neutralises IL-17A and IL-17F, over 48 weeks in patients (pts) with active PsA.

Methods: 206 pts with active PsA, $\geq 3 / 76$ swollen joint count, $\geq 3 / 78$ tender joint count and CASPAR score $>3$, were randomised $(1: 1: 1: 1: 1)$ to receive subcutaneous BKZ 16mg, $160 \mathrm{mg}, 160 \mathrm{mg}$ with $320 \mathrm{mg}$ loading dose (160mg [LD]), 320 mg or placebo (PBO) Q4W, for 12 weeks (double-blind period). After Week 12, pts receiving $\mathrm{PBO}$ or BKZ $16 \mathrm{mg}$ were re-randomised $(1: 1)$ to $\mathrm{BKZ} 160 \mathrm{mg}$ or $320 \mathrm{mg}$ all other pts continued on their initial dose (dose-blind period). The primary endpoint was ACR50 response at Week 12.

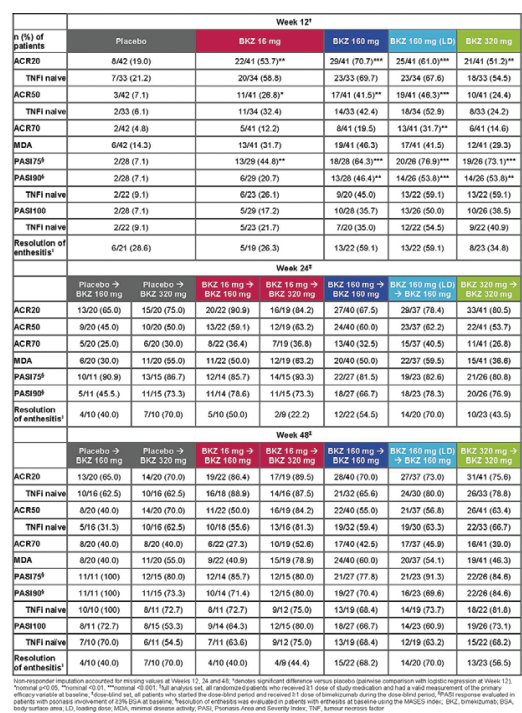

Results: $203 / 206$ and 189/206 pts completed the double- and dose-blind periods, respectively. Overall, demographics and baseline disease characteristics were balanced across groups. $19 \%$ of pts had prior exposure to TNF inhibitors (TNFi). There was a statistically significant $(p<0.05)$ dose-response at Week 12 for ACR50 response rates. At Week 12, significantly more pts receiving BKZ versus PBO achieved ACR50 (primary endpoint: 16-160mg [LD] doses), ACR20 and PASI90 (in those pts with baseline body surface area $\geq 3 \%$; 160-320mg doses) (table). ACR20/50/70, PASI75/90/100, MDA and resolution of enthesitis response rates increased between Week 12 and Week 24 in those continuing on their initial BKZ dose; Week 24 responses were maintained through the study; responses were similar across the three highest dose groups at Week 48 (PASI100 analyses were post hoc). Rapid improvements were observed across all response criteria in pts re-allocated to BKZ 160 or $320 \mathrm{mg}$ (table). BKZ-treated pts naïve to TNF achieved ACR20/50 and PASI90/100 at comparable rates to the overall population at Week 12 and 48. There was no apparent relationship between dose and TEAEs. Serious AEs were reported by $9 / 206$ (4.4\%) pts up to Week 48 (8/206 [3.9\%] patients were receiving BKZ). The most common TEAE up to Week 48 was nasopharyngitis 25/206 [12.1\%]). Oral candidiasis was reported at Week 48 by $10 / 206(4.9 \%)$ pts (all cases during BKZ treatment). No deaths, or cases of IBD or MACE were reported.

Conclusion: Dual neutralisation of IL-17A and IL-17F with BKZ provided substantial improvements in both musculoskeletal and skin outcomes; response rates increased after Week 12 (primary analysis) and were sustained from Week 24 to 48 , with a safety profile consistent with previous BKZ studies. These data provide further support that neutralising IL-17F in addition to IL-17A with BKZ is a promising therapeutic approach in pts with active PsA.

Acknowledgement: Funded by UCB Pharma.

Disclosure of Interests: Christopher T. Ritchlin Grant/research support from: AbbVie, Amgen, UCB Pharma, Consultant for: AbbVie, Amgen, Lilly, Novartis, Pfizer, UCB Pharma, Arthur Kavanaugh Grant/research support from: UCB Pharma, Joseph F. Merola Consultant for: Biogen IDEC, Abbvie, Amgen, Eli Lilly and Company, Novartis, Pfizer, Janssen, UCB, Samumed, Celgene, Sanofi Regeneron, Merck, and GSK, Georg Schett: None declared, Jose U. Scher Consultant for: BMS, Janssen, Novartis, UCB Pharma, Richard B. Warren Grant/ research support from: AbbVie, Almirall, Amgen, Celgene, Janssen, Lilly, LEO, Novartis, Pfizer, UCB Pharma, Consultant for: AbbVie, Almirall, Amgen, Boehringer-Ingleheim, Celgene, Janssen, LEO, Lilly, Novartis, Pfizer, Sanofi, UCB Xenoport, Deepak Assudani Shareholder of: UCB Pharma, Employee of: UCB Pharma, Thomas Kumke Employee of: UCB Pharma, Barbara Ink Shareholder of: GSK, UCB Pharma, Employee of: UCB Pharma, lain Mclnnes Grant/research 
support from: AstraZeneca, Celgene, Compugen, Novartis, Roche, UCB Pharma, Consultant for: AbbVie, Celgene, Galvani, Lilly, Novartis, Pfizer, UCB Pharma DOI: 10.1136/annrheumdis-2019-eular.4883

\section{OP0109 EFFICACY OF FILGOTINIB VS PLACEBO IN ACTIVE PSORIATIC ARTHRITIS: PATIENT-LEVEL DATA FROM EQUATOR, A RANDOMIZED, PHASE 2 STUDY}

Philip J Mease ${ }^{1}$, Dafna D Gladman², Filip van den Bosch ${ }^{3}$, Mykola Stanislavchuk ${ }^{4}$ Anna Rychlewska-Hanczewska ${ }^{5}$, Chantal Tasset ${ }^{6}$, Luc Meuleners ${ }^{6}$,

Robin Besuyen ${ }^{7}$, Jingjing Gao ${ }^{8}$, Mona Trivedi ${ }^{8}$, Laura C Coates ${ }^{9}$, Philip Helliwell ${ }^{10}$ ${ }^{1}$ Swedish Medical Center/Providence St Joseph Health and University of

Washington, Seattle, United States of America; ${ }^{2}$ University of Toronto and Krembil Research Institute, Toronto Western Hospital, Toronto, Canada; ${ }^{3}$ Ghent University Hospital, Ghent, Belgium; ${ }^{4}$ National Pirogov Memorial Medical University, Vinnytsya, Ukraine; ${ }^{5}$ Ai Centrum Medyczne, Poznan, Poland; ${ }^{6}$ Galapagos NV, Mechelen, Belgium; ${ }^{7}$ Galapagos BV, Leiden, Netherlands; ${ }^{8}$ Gilead Sciences, Inc., Foster City, United States of America; ${ }^{9}$ University of Oxford, Oxford, United Kingdom; ${ }^{10}$ Leeds Institute of Rheumatic and Musculoskeletal Medicine, Leeds, United Kingdom

Background: Filgotinib (FIL) is an oral, selective Janus kinase 1 inhibitor in development for the treatment of several inflammatory diseases. In the phase 2 EQUATOR trial (NCT03101670), FIL was efficacious vs placebo (PBO) in patients with active psoriatic arthritis (PsA), and was well tolerated [1]

Objectives: To evaluate the onset and maintenance of response to FIL vs PBO in EQUATOR by evaluating patient-level response over time.

Methods: EQUATOR was a 16-week, multicenter, double-blind study in which patients with active PSA were randomized 1:1 to FIL $200 \mathrm{mg}$ or PBO once daily [1]. Disease activity was assessed at screening, day 1 and weeks 1, 2, 4, 8, 12 and 16 , and the primary efficacy endpoint was the proportion of patients achieving $20 \%$ American College of Rheumatology (ACR20) response. The onset of response was assessed by calculating the median time to ACR20 response using the Kaplan-Meier method and compared between FIL and PBO using the log-rank test. Maintenance of response was assessed by analysing ACR20 response patterns over time in the FIL and PBO groups.

Results: Of 131 patients randomized (FIL: $n=65$; PBO: $n=66), 124$ (95\%) completed the study. Demographics and baseline disease characteristics were similar between groups. The onset of response to FIL was early, with a median (95\% confidence interval) time to first ACR20 response of 4.07 weeks $(2.29,4.14)$ in the FIL group compared with 12.29 weeks $(12$, not reached) in the $\mathrm{PBO}$ group $(\mathrm{p}<0.0001$; Figure 1). ACR20 responses were achieved at week 16 in $80.0 \%(52 / 65)$ and $33.3 \%(22 / 66)$ of patients in the FIL and PBO groups, respectively, using the nonresponder imputation method, and $86.7 \%(52 / 60)$ and $34.4 \%(22 / 64)$, respectively, using observed cases. The number of patients who presented with a stable ACR20 response (i.e. the response was maintained once initially achieved regardless of the time point at which the patient first became a responder) among those who were responders at week 16 (i.e. the primary endpoint) was higher in the FIL group than in the PBO group (80.8\% [42/52] vs 68.2\% [15/22]) (Figure 2). Similar trends were observed for other efficacy endpoints representing various manifestations of PsA.

Conclusion: In general, patients treated with FIL achieved an ACR20 response earlier than those on PBO and these responses appeared to be more stable. In
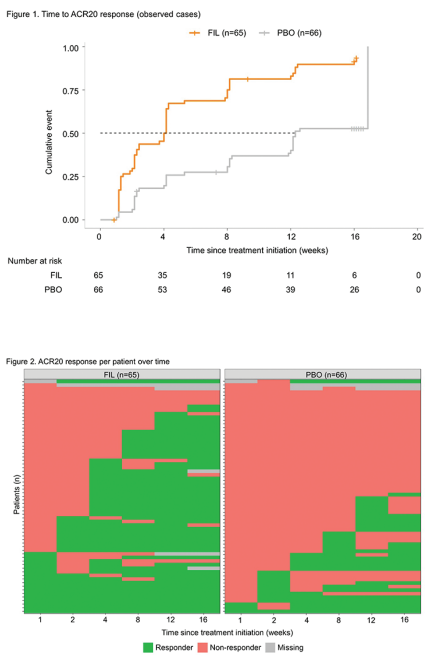

the PBO group, there were more occurrences of the response being lost over time and fewer cases of regaining a lost response.

\section{REFERENCE:}

[1] Mease P, et al. Lancet 2018;392:2367-77.

Acknowledgement: This study was funded by Galapagos NV (Mechelen, Belgium). Medical writing support was provided by Alice Wareham PhD, CMPP (Aspire Scientific Ltd, Bollington, UK) and funded by Galapagos NV.

Disclosure of Interests: Philip J Mease Grant/research support from: AbbVie, Amgen, BMS, Celgene, Janssen, Lilly, Novartis, Pfizer, SUN and UCB, Consultant for: AbbVie, Amgen, BMS, Galapagos, Gilead Sciences, Inc., Janssen, Lilly, Novartis, Pfizer, SUN and UCB, Speakers bureau: AbbVie, Amgen, BMS, Celgene, Genentech, Janssen, Lilly, Novartis, Pfizer and UCB, Dafna D Gladman Grant/research support from: AbbVie, Amgen, Celgene, Lilly, Novartis, Pfizer and UCB, Consultant for: AbbVie, Amgen, BMS, Celgene, Galapagos, Gilead, Janssen, Lilly, Novartis, Pfizer, and UCB, Filip van den Bosch Consultant for: AbbVie, BMS, Galapagos, Janssen, Lilly, Merck, Novartis, Pfizer and UCB, Speakers bureau: AbbVie, BMS, Janssen, Lilly, Merck, Novartis, Pfizer and UCB., Mykola Stanislavchuk Grant/research support from: AstraZeneca, Celltrion, Galapagos Genentech, GlaxoSmithKline, Human Genome, Lilly, Medlmmune, Pfizer, Roche and UCB, Anna Rychlewska-Hanczewska Grant/research support from: Galapagos and Gilead Sciences, Inc., Chantal Tasset Shareholder of: Warrants from Galapagos, Employee of: Galapagos, Luc Meuleners Shareholder of: Warrants from Galapagos, Employee of: Galapagos, Robin Besuyen Shareholder of: Warrants from Galapagos, Employee of: Galapagos, Jingjing Gao Shareholder of: AbbVie and Gilead Sciences, Inc., Employee of: Gilead Sciences, Inc., Mona Triv edi Shareholder of: Amgen and Gilead Sciences, Inc., Employee of: Gilead Sciences, Inc., Laura C Coates Grant/research support from: AbbVie, Celgene, Lilly, Novartis and Pfizer, Consultant for: AbbVie, Amgen, BMS, Celgene, Galapagos, Gilead Sciences Inc., Janssen, Lilly, Novartis, Pfizer, Prothena Corp and UCB, Philip Helliwell Grant/research support from: Paid to charity: from AbbVie, Jans sen and Novartis, Consultant for: Paid to charity: from AbbVie, Amgen, Pfizer, and UCB and Celgene. Paid to self: from Celgene and Galapagos

DOI: 10.1136/annrheumdis-2019-eular.6064

\section{OP0110 \\ IXEKIZUMAB IMPROVES SIGNS AND SYMPTOMS OF PSORIATIC ARTHRITIS IN PATIENTS WHO HAVE HAD INADEQUATE RESPONSE TO 1 OR 2 TUMOR NECROSIS FACTOR INHIBITORS}

L Bruce Kirkham ${ }^{1}$, Carlos Sesin ${ }^{2}$, Aubrey Trevelin Sprabery ${ }^{3}$, Chen-Yen Lin ${ }^{3}$ Amanda M. Gellett ${ }^{3}$, Anthony Turkiewicz ${ }^{4} .{ }^{1}$ Guy's Hospital, London, United Kingdom; ${ }^{2}$ Mount Sinai Medical Center, Miami, United States of America; ${ }^{3}$ Eli Lilly and Company, Indianapolis, United States of America; ${ }^{4}$ Rheumatology Associates, Birmingham, United States of America

Background: Psoriatic arthritis (PsA) is a progressive, chronic inflammatory dis ease often treated with tumor necrosis factor inhibitors (TNFi) when conventional treatments fail. Patients with inadequate response to TNFi represent a more difficult-to-treat population.

Objectives: To report the efficacy of ixekizumab (IXE), a monoclonal antibody that selectively targets interleukin-17A, in patients with inadequate response to 1 TNFi or 2 TNFi.

Methods: In a Phase 3 study (SPIRIT-P2; NCT02349295), patients who had an inadequate response or intolerance to 1 or $2 \mathrm{TNFi}$ were randomized to receive subcutaneous IXE $80 \mathrm{mg}$ every 2 weeks (IXEQ2W; $\mathrm{N}=123$ ) or every 4 weeks (IXEQ4W; $\mathrm{N}=122$ ), after a 160-mg starting dose, or placebo (PBO; $\mathrm{N}=118$ ) for up to 24 weeks. At Week 16, patients not meeting predefined criteria $(<20 \%$ improve ment in tender joint count [TJC] and swollen joint count [SJC]) received rescue therapy and were imputed as nonresponders at Weeks 20 and 24. At Week 24, PBO patients were rerandomized to IXEQ2W or IXEQ4W through Week 52 and excluded from the 52-week analysis. At Week 32 or any subsequent visit, patients were discontinued if they did not reach $\geq 20 \%$ improvement from baseline in both TJC and SJC. These ad-hoc data were derived from patients in the intent-to-treat population with prior inadequate response to TNFi; intolerant patients were excluded from the analysis. Efficacy was measured by percentage of patients who attained $\geq 50 \%$ improvement in American College of Rheumatology response criteria (ACR50), an improvement in Health Assessment QuestionnaireDisability Index (HAQ-DI) $\geq 0.35$, minimal disease activity (MDA), Disease Activity Score 28 - C-reactive protein (DAS28-CRP) EULAR Good Response criteria, and Disease Activity in Psoriatic Arthritis (DAPSA) $\leq 14$.

Results: At baseline, 1-TNFi inadequate responders were, on average, 52 years of age with a PsA diagnosis for 10 years; $40 \%$ were using MTX, and HAQ-DI was 1.2. 2-TNFi inadequate responders were 52 years of age with a PsA diagnosis for 11 years; $42 \%$ were using MTX, and HAQ-DI was 1.3. Regardless of inadequate response to 1 or $2 \mathrm{TNFi}$, at Week 24 significantly more patients receiving Q4W or 